\title{
Applications of a Theorem on Partitioned Matrices
}

\author{
Emilie V. Haynsworth
}

(May 25, 1959)

\begin{abstract}
A reduction formula for partitioned matrices is applied to block-stochastic matrices and other types of partitioned matrices in order to reduce the computation in finding the eigenvalues. Such matrices occur frequently in physics and chemistry, and the reduction formulas given here have been applied successfully in practical problems. In addition, some results of $\mathrm{A}$. Brauer on stochastic matrices and of $\mathrm{J}$. Williamson in partitioned matrices are generalized.
\end{abstract}

\section{Introduction}

In a previous paper, ${ }^{1}$ a general reduction formula was given for certain partitioned matrices,

$$
A=\left(\begin{array}{cccc}
A_{11} & A_{12} & \cdots & A_{1 t} \\
A_{21} & A_{22} & \cdots & A_{2 t} \\
\cdot & \cdot & \cdot \\
\cdot & \cdot & \cdot \\
\cdot & \cdot & \cdot \\
A_{t 1} & A_{t 2} & \cdots & A_{t t}
\end{array}\right)
$$

of order $N$, where the submatrices, $A_{i j}$, have dimensions $n_{i} \times n_{j}, i, j=1,2, \ldots t$, and

$$
\sum_{i=1}^{t} n_{i}=N \text {. }
$$

One of the special cases of this general theorem is of particular interest in practical applications to the problem of finding the eigenvalues of a matrix and, in fact, has been applied successfully to such problems. This special case is given below as theorem 1, since its proof is simpler than that for the general theorem and exhibits the transformation matrices needed for the reduction formula.

This result is used in section 3 to give generalizations of some theorems by A. Brauer ${ }^{2}$ on stochastic matrices. Other applications are given in later sections.

As in the previous paper, for a given partitioning of a matrix $A$ we shall call the submatrices, $A_{i j}$, the blocks of $A$ and we shall write $A=\left(A_{i j}\right)$. The elements of the blocks will be denoted by $a_{h k}^{(i j)}$, i.e.,

$$
A_{i j}=\left(a_{\substack{(i j) \\ k}}^{(i)}\right. \text {. }
$$

Unless otherwise stated, the matrices will be

${ }^{1}$ E. Haynsworth, Reduction formulas for partitioned matrices (to be published).

${ }_{2}$ A. Brauer, Limits for the characteristic roots of a matrix, IV, Duke Math. J. $19,75(1952)$. arbitrary complex matrices. Also, since we will be dealing throughout with matrices $A=\left(A_{i j}\right)$ of form (1), we will assume, unless otherwise indicated, that the statements and formulas given are true for $i, j=1,2, \ldots, t$.

\section{Special Reduction Formula}

For the sake of completeness we include a lemma from the previous paper ${ }^{1}$ which is needed in the proofs of theorems 1 and 3 . In this lemma we consider three cases where there are zeros in convenient places in the blocks of a partitioned matrix, $A$. In each of these cases $A$ is reducible, and the proof consists merely in defining the permutation matrix which puts $A$ into the reduced form,

$$
\widetilde{A}=\left(\begin{array}{ll}
C & O \\
D & T
\end{array}\right),
$$

where $C$ and $T$ are square matrices and $O$ is a matrix composed entirely of zeros.

Lemma: Given a partitioned matrix $A$ of order $N$ with $n_{1} \times n_{j}$ blocks $A_{i j}$ :

1. If $n_{i}=n \quad(i=1,2, \ldots, \mathrm{t})$ and the blocks $A_{i j}$ are lower triangular with elements $\lambda_{i j}^{(h)}, h=1,2, \dot{\tilde{A}} . ., n$, on the diagonal, $A$ is similar to a matrix $\tilde{A}$, with blocks $\tilde{A}_{h h}=\left(\lambda_{i j}^{(\mathrm{h})}\right), \quad h=1,2, \ldots ., n$, on the diagonal, and zero blocks above the diagonal (this case is given by Williamson ${ }^{3}$ ).

2. If

$$
A_{i j}=\left(\begin{array}{ll}
C_{i j} & O \\
D_{i j} & T_{i j}
\end{array}\right),
$$

where all matrices $T_{i j}$ are square, of order $r$, and the matrices $C_{i j}$ are $\left(n_{i}-r\right) \times\left(n_{j}-r\right)$, then $A$ has the form (2), where $C=\left(C_{i j}\right)$ and $T=\left(T_{i j}\right)$.

3. If $A_{i j}$ has the form (3) where the matrices $T_{i j}$ are lower triangular, we will say $A_{i j}$ is partially triangular. Then if $A$ has blocks $A_{i j}$ which are all partially trianqular with submatrices, $T_{i j}$ of order $r$, having elements $t_{i j}^{(h)}, h=1,2, \ldots ., r$, on the diagonal, $t r$ roots of $A$ are roots of the $r$ matrices, $\left(t_{i j}^{(h)}\right)$.

${ }^{3} \mathrm{~J}$. Williamson, The latent roots of a matrix of special type, Bull. Am. Math. Soc. $\mathbf{3 7}, 585$ (1931). 
The proofs given below would follow in a corresponding manner if all blocks were transposed.

\section{Proof:}

1. The rows and columns of $\mathbf{A}$ should be arranged in the order

$$
\begin{gathered}
1, n+1,2 n+1, \ldots,(t-1) n+1 ; \\
2, n+2,2 n+2, \ldots,(t-1) n+2 ; \\
. \quad . \\
n, 2 n, 3 n, \quad \ldots, \quad t n .
\end{gathered}
$$

Then the new matrix $\tilde{A}$ will have the matrices $\widetilde{A}_{h h}=\left(\lambda_{i j}^{(h)}\right)$ on the diagonal and $\widetilde{A}_{h k}=0$ for $i<j$, so its roots are the roots of the $n$ matrices, $\widetilde{A}_{h k}$.

2. Let $\sum_{i=1}^{k} n_{i}=N_{k}$. Then $N_{1}=n_{1}, N_{t}=N$. If we arrange the rows and columns of $A$ in the following order:

$$
\begin{gathered}
1,2, \ldots, N_{1}-r, \\
N_{1}+1, N_{1}+2, \ldots, N_{2}-r, \\
. \cdot \\
N_{t-1}+1, N_{t-1}+2, \ldots, N_{t}-r, \\
N_{1}-r+1, N_{1}-r+2, \ldots, N_{1}, \\
. \quad . \\
N_{t}-r+1, N_{t}-r+2, \ldots, N_{t},
\end{gathered}
$$

we have a new matrix $A$ in which the matrices $C_{i j}$ are together in the upper left corner, and the matrices $T_{i j}$ are together in the lower right corner. So $A$ will have the form (2) where $C=\left(C_{i j}\right)$ and $T=\left(T_{i j}\right)$.

3. Case 3 follows immediately now by first applying the permutation in 2 to the rows and columns of $A$ and then applying the permutation in 1 to the rows and columns containing $T$.

Theorem 1: Suppose the blocks $A_{i j}$ of the partitioned matrix given in (1) satisfy the equation

$$
A_{i j} X_{j}=X_{i} B_{i j}
$$

where $B_{i j}$ is a square matrix of order $r, 0<r \leqq n_{i}$, with strict inequality for at least one value of $i$, and $X_{i}$ is an $n_{i} \times r$ matrix with a nonsingular matrix of order $r, X_{l}^{(i)}$, in the first $r$ rows. Let the last $n_{i}-r$ rows of $X_{i}$ be $X_{2}^{(i)}$, and let

$$
A_{i j}=\left(\begin{array}{ll}
A_{11}^{(i j)} & A_{12}^{(i j)} \\
A_{21}^{(i j)} & A_{22}^{(i j)}
\end{array}\right)
$$

where $A_{11}^{(i j)}$ is square, of order $r$. Then $A$ is similar to the matrix,

$$
R=\left(\begin{array}{ll}
B & * \\
O & C
\end{array}\right)
$$

where $B$ is a partitioned matrix of order tr with blocks $B_{i j}$, as defined in $(6)$, and $C$ has blocks,

$$
C_{i j}=\left(A_{22}^{(i j)}-X_{2}^{(i)}\left(X_{1}^{(i)}\right)^{-1} A_{12}^{(i j)}\right)
$$

with dimensions $\left(n_{i}-r\right) \times\left(n_{j}-r\right)$. (If either $n_{i}$ or $n_{j}=r$, the corresponding block $C_{i j}$ does not appear. By hypothesis not all $n_{i}$ are equal to $r$, or else we would be left with the matrix $B=\left(B_{i j}\right)$ which would be similar to $A$.)

Thus the roots of $A$ are the roots of the smaller matrices $B$ and $C$.

Proof: Let $P_{i}$ be a matrix of order $n_{i}$ :

$$
P_{i}=\left(\begin{array}{cc}
X_{1}^{(i)} & 0 \\
X_{2}^{(i)} & I_{n_{i}-r}
\end{array}\right)
$$

then

$$
P_{i}^{-1}=\left(\begin{array}{lr}
\left(X_{1}^{(i)}\right)^{-1} & O \\
-X_{2}^{(i)}\left(X_{1}^{(i)}\right)^{-1} & I_{n_{i}-r}
\end{array}\right)
$$

where $I_{k}$ represents an identity matrix of order $k$.

Since by (7) and (10)

$$
A_{i j} P_{j}=\left(\begin{array}{ll}
{\left[A_{11}^{(i j)} X_{1}^{(j)}+A_{12}^{(i j)} X_{2}^{(j)}\right]} & A_{12}^{(i j)} \\
{\left[A_{21}^{(i j)} X_{1}^{(j)}+A_{22}^{(i j)} X_{2}^{(j)}\right]} & A_{22}^{i j}
\end{array}\right),
$$

then by (6),

$$
A_{i j} P_{j}=\left(\begin{array}{ll}
{\left[X_{1}^{(i)} \cdot B_{i j}\right]} & A_{12}^{(i j)} \\
{\left[X_{2}^{(i)} \cdot B_{i j}\right]} & A_{22}^{(i j)}
\end{array}\right),
$$

and, using (11) and (9),

$$
\tilde{A}_{i j}=P_{i}^{-1} A_{i j} P_{j}=\left(\begin{array}{cc}
B_{i j} & \left(X_{1}^{(i)}\right)^{-1} A_{12}^{(i j)} \\
0 & C_{i j}
\end{array}\right) .
$$

So, if we let $P$ be the direct sum of the matrices $I^{\prime}$,

$$
P=\Sigma \cdot P_{i}=P_{1} \dot{+} P_{2} \dot{+} \ldots \dot{+} P_{\iota},
$$

and let

$$
\tilde{A}=P^{-1} A P,
$$

then $\tilde{A}$ has the blocks $\tilde{A}_{i j}$ given in (12), and the simultaneous permutation of rows and columns of $A$ given by case 2 of the lemma above, will put it into form (8). 


\section{Generalizations of Brauer Theorems on Stochastic Matrices}

A. Brauer ${ }^{2}$ proved a number of interesting theorems about generalized stochastic matrices, i.e., matrices $A=\left(a_{i j}\right)$ of order $n$ such that

$$
\sum_{j=1}^{n} a_{i j}=s, \quad(i=1,2, \ldots n) .
$$

Such matrices have as one root, $s$, and the vector corresponding to this root is

$$
U_{n}=(1,1, \ldots, 1)
$$

The reduction formula of theorem 1 is applied in this section to matrices which can be partitioned into stochastic blocks, to give generalizations of Brauer's results.

If a matrix $A$ of order $N$ can be partitioned into rectangular $\left(n_{i} \times n_{j}\right)$ blocks, in each of which the row-sums are all equal, i.e.,

$$
A_{i j}=\left(a_{h k}^{(i j)}\right), \quad\left(h=1,2, \ldots, n_{i}, \quad k=1,2, \ldots, n_{j}\right)
$$

with

$$
\sum_{k=1}^{n_{i}} a_{h k}^{(i j)}=s_{i j}, \quad\left(h=1,2, \ldots, n_{i}\right),
$$

$A$ is a block-stochastic matrix.

Theorem 2: If $A$ is block-stochastic, (1) troots of $A$ are roots of the matrix $S=\left(s_{i j}\right)$, and (2) the other $N-t$ are roots of the partitioned matrix of order $N-t$,

$$
C=\left(C_{i j}\right) \text {, }
$$

$C_{i j}=\left(a_{h k}^{(i j)}-a_{1 k}^{(i j)}\right), \quad\left(h=2,3, \ldots, n_{j}, \quad k=2,3, \ldots, n_{j}\right)$.

If either $n_{i}$ or $n_{j}$ is 1 , the block $C_{i j}$ is omitted, so $i$ and $j$ do not necessarily take on all values 1,2, . . . , t.

Proof: Since, by (14a) and (15)

$$
A_{i j} U_{n_{i}}=s_{i j} U_{n_{i}}
$$

statement 1 follows immediately, for by (17), if we set $r=1$ in (6) the matrices $B_{i j}$ of theorem 1 are simply the elements $s_{i j}$ given by $(15)$. Then, if we subdivide $A_{i j}$ as in (7), $A_{22}^{(i j)}$ consists of all the elements of the block $A_{i j}$ except for those in the first row and column, i.e.,

$$
A_{22}^{(i j)}=\left(a_{h k}^{(i j)}\right) \quad\left(h=2, \ldots, n_{i} ; \quad k=2, \ldots, n_{j}\right),
$$

and

$$
A_{12}^{(i j)}=\left(a_{1 k}^{(i j)}\right) \quad\left(k=2, \ldots, n_{j}\right) .
$$

Since by (17), the matrices $X_{i}$ in (6) are the vectors $U_{n_{i}}$, we have

$$
X_{1}^{(i)}=1, \quad X_{2}^{(i)}=U_{n_{i}-1} .
$$

Then, since $U_{n_{i}-1} A_{12}^{(i j)}$ is a matrix having in each row the elements

$$
a_{12}^{(i j)}, \ldots, a_{1, n_{j}}^{(\ell j)}
$$

statement 2 follows from (9). For example, the following matrix arose from a problem in molecular physies: ${ }^{4}$

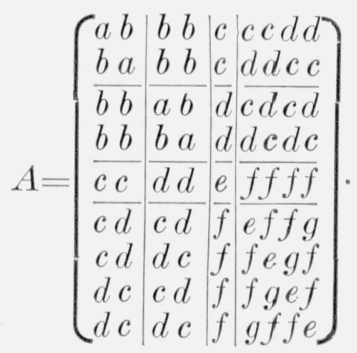

The lines are drawn in to show how $A$ is subdivided into stochastic blocks. Then by theorem 3 , the roots of $A$ are those of the $5 \times 5$ matrix,

$$
C=\left(\begin{array}{ccccc}
a-b & 0 & c-d & c-d & d-c \\
0 & a-b & c-d & d-c & c-d \\
c-d & c-d & e-g & 0 & 0 \\
c-d & 0 & f-g & e-f & g-f \\
0 & c-d & f-g & g-f & e-f
\end{array}\right)
$$

and those of the $4 \times 4$ matrix,

$$
S=\left(\begin{array}{cccc}
a+b & 2 b & c & 2 c+2 d \\
2 b & a+b & d & 2 c+2 d \\
2 c & 2 d & e & 4 f \\
c+d & c+d & f & e+2 f+g
\end{array}\right) .
$$

It is easy to see that $C$ can again be divided into stochastic blocks where $n_{1}=2, \quad n_{2}=1, \quad n_{3}=2$. So the five roots of $C$ are those of the $2 \times 2$ matrix,

$$
C_{1}=\left(\begin{array}{cc}
a-b & 2 c-2 d \\
c-d & e-g
\end{array}\right)
$$

and the $3 \times 3$ matrix

$$
C_{2}=\left(\begin{array}{ccc}
a-b & c-d & 0 \\
2 c-2 d & e-g & 0 \\
c-d & f-g & e-2 f+g
\end{array}\right) .
$$

Thus $e-2 f+g$ is a root, and there are two degenerate roots since the matrix $C_{1}$ corresponds to the quadratic matrix which remains when the root $e-2 f+g$ is factored out of $C_{2}$.

Corollary (Theorem 2): If $A$ is a block-stochastic matrix with $n_{i} \times n_{j}$ blocks $A_{i j}$, and $P=\sum \cdot P_{i}$, $Q=\sum \cdot Q_{i}$ where $P_{i}$ and $Q_{i}$ are permutation matrices of

\footnotetext{
4 The author is grateful to J. Bradley for supplying this example.
} 
order $n_{i}$, any matrix $P A Q$ is also block-stochastic and has $t$ roots in common with $A$.

Proof: Since the row-sums in a given block would be unchanged by permutations within the block, this follows immediately from statement 1 of theorem 2 .

Brauer (see footnote 2) proved the following theorems for certain special stochastic matrices:

1. Assume that

$$
a_{i j}=c_{j} \quad(j=2,3, \ldots n ; \quad i<j) .
$$

Then $A$ has the same roots as the diagonal matrix

$$
B=\operatorname{diag}\left(a_{22}-c_{2}, a_{33}-c_{3}, \ldots, a_{n n}-c_{n}, s\right) .
$$

2. Assume

$a_{i j}=c, j \neq i$, and $a_{i j}=b$

$$
\left(j=j_{1}, j_{2}, \ldots, j_{r}\right)
$$

Then $(b-c)$ is a root of multiplicity $r$.

We generalize these results in the following theorem for block-stochastic matrices:

Theorem 3: Let A be a block-stochastic matrix with submatrices $A_{i j}=\left(a_{h k}^{i j}\right)$ having constants $c_{k}$ above the diagonal in the last $r$ columns of each block, $0<r<\min n_{i}$, i.e.,

$$
a_{h k}^{(i j)}=c_{k}^{(i j)} \quad\left(k>n_{n j}-r ; \quad h<k+n_{i}-n_{j}\right) .
$$

Then $\operatorname{tr}$ of the roots of $A$ are roots of the matrices

$$
Q_{k k}=\left(a_{k k}^{(i j)}-c_{k}^{i j}\right) \quad\left(k=n_{j}-r+1, \ldots, n_{j}\right) .
$$

(The second of Brauer's theorems comes as a special case of the above if we permute the rows and columns so that the columns $j_{1}, j_{2}, \ldots, j_{r}$ are the last $r$ columns of the matrix. Also it isn't necessary that all the nondiagonal elements of a column be equal as in Brauer's hypothesis.)

Proof: By theorem 2, $N$ - $t$ roots of $A$ are roots of $C=\left(C_{i j}\right)$. Here we have $i, j=1,2, \ldots, t$, since by hypothesis, $0<r<\min _{i} n_{i}$, and thus $\min _{i} n_{i}>1$.

But by (16) and (18), $C_{i j}$ is a partially triangular matrix with elements $a_{k k}^{(i j)}-c_{k}^{(i j)}$ on the diagonal of the triangular portion, i.e., for $k=n_{j}-r+1, \ldots, n_{j}$. Then, using case 3 of the lemma above, we can permute the rows and columns of $\tilde{\mathrm{A}}$ so that $\tilde{\mathrm{A}}$ is similar to the matrix

$$
R=\left(\begin{array}{ll}
P & * \\
O & Q
\end{array}\right)
$$

when $Q=\left(Q_{h k}\right)$ is a matrix of order $t r$ having square blocks of order $t$, defined by (15), on the diagonal, and $Q_{h k}=0$ for $k<h$.

\section{Other Applications}

In this section we will mention several other types of partitioned matrices, $A=\left(A_{i j}\right)$, such that

$$
P A P^{-1}=\left(\begin{array}{ll}
B & * \\
O & C
\end{array}\right),
$$

where $P$ is the direct sum of transformation matrices $P_{i}$, and $B=\left(B_{i j}\right), C=\left(C_{i j}\right)$, are partitioned matrices which will be defined in each case. We also give a case where the transformation $P A P^{-1}$ produces a real matrix from a complex one.

Since the proof in each case is the same and consists merely in performing the indicated matrix multiplications, it will not be given in detail.

\subsection{Block-Circulant Matrices}

This is the case where each block $A_{i j}$ is a circulant matrix of order $n$. The result (21) is given by Williamson. ${ }^{3}$

The roots of $A$ are roots of the $n$ matrices of order $t$,

$$
\left(\lambda_{i j}^{h}\right)=\left(\sum_{k=1}^{n} a_{h k}^{(i j)} \epsilon_{h}^{k}\right) \quad(h=1,2, \ldots, n)
$$

where $\epsilon_{h}$ is one of the $n$th roots of unity.

\subsection{Block-Singular Matrices}

If $A$ is singular, of rank $n-1$, there exists a vector $x=\left(x_{1}, x_{2}, \ldots, x_{n}\right)$, with components not all zero, such that

$$
A X=X \cdot O \text {. }
$$

We may assume, without loss of generality, that $x_{1}=1$. Then, as in theorem 1 , if $X=1+X_{2}$, we can let

$$
P=\left(\begin{array}{cc}
1 & 0 \\
-X_{2} & I_{n-1}
\end{array}\right)
$$

and, applying the transformation (16), we can show that the nonzero roots of $A$ are roots of

$$
C=\left(a_{i j}-x_{i} a_{i j}\right) \quad(i, j=2, \ldots, n) .
$$

If $A$ has rank less than $n-1$, the same type of transformation can be applied to the matrix $C$ and this can be continued until a nonsingular matrix is obtained. Or, we may be able to partition $A$ into blocks so that

$$
A_{i j} X_{j}=X_{i} \cdot O
$$

Then if we let $P=\sum P_{i}$, where each $P_{i}$ has the form (22), and apply transformation (20), we show 
that the roots of $A$ are the roots of

and

$$
B=\left(B_{i j}\right)=(O) \text { oî order } t
$$

where

$$
C=\left(C_{i j}\right)
$$

$$
\begin{aligned}
& C_{i j}=\left(a_{h k}^{(i j)}-x_{h}^{(i)} a_{1 k}^{(i j)}\right) \\
&\left(h=2, \ldots, n_{i} ; \quad k=2, \ldots, n_{j}\right) .
\end{aligned}
$$

Let

\subsection{Block Tridiagonal Matrices}

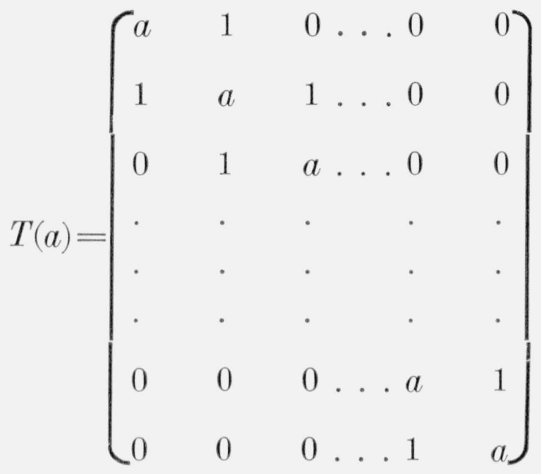

It is well known $n^{5}$ that such matrices have characteristic roots which may be written in the form

$$
\lambda_{k}=a+2 \cos (k \pi / n+1) \quad(k=1,2, \ldots, n)
$$

and, if we let $\theta_{k}=k \pi / n+1$ it is easy to show that the characteristic vector corresponding to $\lambda_{k}$ has components

$$
\left(\sin \theta_{k}, \sin 2 \theta_{k}, \ldots, \sin n \theta_{k}\right)
$$

since the trigonometric identities

$$
a \sin \theta_{k}+\sin 2 \theta_{k}=\left(a+2 \cos \theta_{k}\right) \sin \theta_{k},
$$

$\sin (m-1) \theta_{k}+a \sin m \theta_{k}+\sin (m+1) \theta_{k}$

$$
=\left(a+2 \cos \theta_{k}\right) \sin m \theta_{k} \quad(m=2, \ldots, n-1),
$$

$\sin (n-1) \theta_{k}+a \sin n \theta_{k}=\left(a+2 \cos \theta_{k}\right) \sin n \theta_{i}$

hold for each $\theta_{k}$ as defined above.

So if $A=\left(A_{i j}\right)$ where the blocks $A_{i j}$ are all tridiagonal,

$$
A_{i j}=T\left(a_{i j}\right),
$$

these blocks can be simultaneously reduced to triangular form using the matrix of characteristic vectors (25), since these are independent of the elements $a_{i j}$. Thus, according to a theorem by Williamson, ${ }^{4}$ the roots of $A$ are those of the $n$ matrices,

$$
\begin{array}{r}
\left(a_{i j}+2 \cos (\pi / n+1)\right), \ldots, \\
\left(a_{i j}+2 \cos (n \pi / n+1)\right) .
\end{array}
$$

${ }^{5}$ D. E. Rutherford, Some continuant determinants arising in physics and chemistry, II, Proc. Roy. Soc. Edinburgh 63, III (1952).
If, in addition, the matrix $\left(a_{i j}\right)$ is stochastic, with row sum $s$, by theorem $1, A$ has as $n$ of its roots the number $s+(1 / n+1) 2 t \cos k \pi(k=1, \ldots, n)$. The other $n(t-1)$ roots are degenerate with multiplicity $n$, since the matrices $C_{i j}$ in (5) become, by (22) for any value of $n$,

$$
\left(a_{i j}-a_{1 j}\right) \quad(i, j=2, \ldots t) .
$$

\subsection{Complex Matrices Similar to Real Matrices}

$A=\left(A_{i j}\right)$ where each block is a $2 \times 2$ matrix defined as follows:

$$
A_{i i}=\left(\begin{array}{cc}
a_{i}+\sqrt{-1} b_{i} & 0 \\
0 & a_{i}-\sqrt{-1} b_{i}
\end{array}\right) \quad(i=1, \ldots, k) .
$$

(We use $\sqrt{-1}$ instead of $i$ to avoid confusion with the subscript.)

$A_{i j}=\left(\begin{array}{cc}a_{i j} & b_{i j} \\ b_{i j} & a_{i j}\end{array}\right) \quad(i, j=1,2, \ldots, k ; \quad i \pm j)$

$A_{i j}=\left(\begin{array}{cc}a_{i j} & b_{i j} \\ a_{i j} & b_{i j}\end{array}\right) \quad(i=1,2, \ldots, k ; \quad j=k+1, \ldots, t)$

$A_{i j}=\left(\begin{array}{cc}a_{i j} & a_{i j} \\ b_{i j} & b_{i j}\end{array}\right) \quad(i=k+1, \ldots, t ; \quad j=1, \ldots, k)$

and $A_{i j}$ an arbitrary real matrix $(i, j=k+1, \ldots, t)$. Then,

$P_{i}=\left(\begin{array}{cc}\frac{1+\sqrt{-1}}{2} & \frac{1-\sqrt{-1}}{2} \\ \frac{1-\sqrt{-1}}{2} & \frac{1+\sqrt{-1}}{2}\end{array}\right) \quad(i=1,2, \ldots, k)$,

$P_{i}=I_{2} \quad(i=k+1, \ldots, n)$.

$$
\begin{aligned}
P_{i} A_{i i} P_{i}^{-1}=\left(\begin{array}{rr}
a & -b \\
b & a
\end{array}\right) \quad(i=1, \ldots, k), \\
P_{i} A_{i j} P_{j}^{-1}=A_{i j}, \quad(i, j=1, \ldots, k ;), \\
P_{i} A_{i j}=A_{i j} \quad(i=1, \ldots, k, \quad j=k=1, \ldots, t), \\
A_{i j} P_{j}^{-1}=A_{i j} \quad(i=k+1, \ldots, t ; \quad j=1, \ldots k),
\end{aligned}
$$

Since

$$
\widetilde{A}=P A P^{-1}
$$


has the same elements as $A$ except in the $2 \times 2$ blocks on the diagonal for $i=1,2, \ldots, k$, and the elements in these blocks are real. (Matrices of this type also arose from an applied problem at the National Bureau of Standards.)

\section{Applications to Determinants and Systems of Equations}

The transformations given here for a partitioned matrix $A=\left(A_{i j}\right)$ can obviously be applied to finding the determinant of such a matrix since if

$$
\begin{gathered}
\tilde{A}=P A P^{-1}=\left(\begin{array}{ll}
B & D \\
O & C
\end{array}\right), \\
|\tilde{A}|=|A|=|B| \cdot|C| .
\end{gathered}
$$

These transformations could also be profitably applied to the solution of a system of linear equations with coefficient matrix $A$; i.e., the system of equations

$$
A X=K
$$

is, by (29), the same as

$$
\left(P^{-1} \tilde{A} P\right) X=K
$$

or

where

$$
\tilde{A} \tilde{X}=\tilde{K}
$$

and

$$
P K=\tilde{K}
$$

$$
P X=\tilde{X} \text {. }
$$

Then if $X=X_{1}+X_{2}$ and $K=K_{1}+K_{2}$ are partitioned to correspond with the dimensions of $B$ and $C$, we can first solve the system

$$
C \tilde{X}_{2}=\tilde{K}_{2}
$$

and then the system

$$
B \tilde{X}_{1}=\tilde{K}_{1}-D \tilde{X}_{2}
$$

So, solving these two systems of linear equations and the comparatively simple system (31) (which can be partitioned as $P$ is partitioned), we have the solution of (29).

Such a transformation would be especially valuable in solving a large system on an electronic computer, where the length of time for solution goes up on the order of $n^{3}$. Thus, if we cut the dimensions in half by transformation (29), systems (32) and (33) would each take one-eighth as long to solve as the original system, and if we assumed only that $P$ could be partitioned into two parts the total solution time would be at most one-half that of the original system. In many cases the time could be cut to approximately one-fourth or less.

WAShington, D.C.

(Paper 63B2-9) 